\title{
Stability analysis of initial value problem of pantograph-type implicit fractional differential equations with impulsive conditions
}

\author{
Arshad Ali ${ }^{1}$, Ibrahim Mahariq2 ${ }^{2^{*}}$ (D), Kamal Shah', Thabet Abdeljawad ${ }^{3,4,5^{*}}$ (D) and Bahaa Al-Sheikh 2,6
}

"Correspondence:

ibmahariq@gmail.com;

tabdeljawad@psu.edu.sa

${ }^{2}$ College of Engineering and

Technology, American University of

the Middle East, Egaila, Kuwait

${ }^{3}$ Department of Mathematics and

General Sciences, Prince Sultan

University, Riyadh, Saudi Arabia

Full list of author information is

available at the end of the article

\section{Springer}

\begin{abstract}
In this paper, we study an initial value problem for a class of impulsive implicit-type fractional differential equations (FDEs) with proportional delay terms. Schaefer's fixed point theorem and Banach's contraction principle are the key tools in obtaining the required results. We apply our results to a numerical problem for demonstration purpose.
\end{abstract}

MSC: Primary 26A33; secondary 34A07; 35B40

Keywords: Pantograph differential equations; Initial value problem; Impulsive condition; Hyers-Ulam stability

\section{Introduction}

Differential equations have been proved to be powerful tools to describe many phenomena in real-world problems. There has been a significant advancement in studying various classes of differential equations. We refer to some recent work [1-5]. In the recent years the trend is changed from classical integer-order derivatives to fractional-order derivatives. This is because of the fact that the study of many mathematical models of realworld problems with fractional-order derivatives produces significant results. The main advantage of noninteger-order derivatives is that they are global operators and produce accurate and stable results, whereas integer-order derivatives are local operators. Due to these dominant advantages, various classes of differential equations are reformulated and constructed in terms of fractional-order derivatives. We refer to some problems such as world population growth models, blood alcohol level problems, video tape models, and so on, where fractional-order derivatives are applied as powerful tools. Similarly, fractionalorder derivatives have applications in other fields like electrodynamics, fluid dynamics, fluid mechanics, and so on; see [6-15].

The class of implicit differential equations is one of the major classes of differential equations. These equations have applications in managerial and economic sciences. In economical problems the differential equations in equilibrium state we dealing with are

(c) The Author(s) 2021. This article is licensed under a Creative Commons Attribution 4.0 International License, which permits use sharing, adaptation, distribution and reproduction in any medium or format, as long as you give appropriate credit to the original author(s) and the source, provide a link to the Creative Commons licence, and indicate if changes were made. The images or other third party material in this article are included in the article's Creative Commons licence, unless indicated otherwise in a credit line to the material. If material is not included in the article's Creative Commons licence and your intended use is not permitted by statutory regulation or exceeds the permitted use, you will need to obtain permission directly from the copyright holder. To view a copy of this licence, visit http://creativecommons.org/licenses/by/4.0/ 
mostly of implicit type. Similarly, with the help of implicit functions, we can investigate noteworthy features of most real-life isolines or surface geometry. We refer to some recent work on implicit differential equations [16-18].

On other hand, differential equations with impulsive conditions play an important role almost in every subject of science. Dynamical systems with impulsive phenomena have applications in physics, biology, economics, engineering, and so forth. Differential equations with impulsive conditions are used to model certain processes with discontinuous jumps and abrupt changes that cannot be modeled by classical differential equations; see [19-26]. Considerable attention has been given to impulsive differential equations, but it is worth noticing that many aspects of these equations yet need to be studied and explored.

There are several types of delay differential equations. One type is proportional delay differential equations, also known as pantograph differential equations. The importance of these equations is due to their ability to model several problems in economics, chemistry, medicine, biology, infectious diseases, physiological and pharmaceutical kinetics, chemical kinetics, absorption of light by the interstellar matter, physics, population studies, number theory, the navigational control of ships and aircraft, electronic systems, electrodynamics, quantum mechanics, and so on [27-30].

Implicit differential equations with proportional delay occur in many applied physical applications. In economics the sudden rise and fall in stock exchange or in the status at time $t$ as a function of that time with some delay is inevitable in decision making problems. This is a practical significance of pantograph implicit impulsive differential equations. For some interesting applications of proportional delay differential equations, we refer to [31] and references therein.

Motivated by the aforementioned applications of implicit impulsive pantograph differential equations, in this paper, we study two important aspects; the existence of solutions and the Hyers-Ulam stability of the following initial value problem of implicit impulsive differential equations with proportional delay term:

$$
\left\{\begin{array}{l}
{ }_{0}^{c} D_{x_{n}}^{\alpha} z(x)=f\left(x, z(x), z(m x),{ }_{0}^{c} D_{x_{n}}^{\alpha} z(x)\right), \quad x \in J, \\
x \neq x_{n} \quad \text { for } n=1,2, \ldots, k, 0<\alpha \leq 1,0<m<1, \\
z(0)=z_{0}, \\
\Delta z\left(x_{n}\right)=I_{n}\left(z\left(x_{n}\right)\right), \quad n=1,2, \ldots, k,
\end{array}\right.
$$

where $J=[0, T], T>0,{ }_{0}^{c} D_{x_{n}}^{\alpha}$ denotes the Caputo derivative at points other than $x_{n}$ in $J$, and $f: J \times R^{3} \rightarrow R$ and $\Phi: C(J, R) \rightarrow R$ are given continuous functions. Further, $I_{n}: R \rightarrow R$ are nonlinear impulsive mappings, and $\Delta z\left(x_{n}\right)=z\left(x_{n}^{+}\right)-z\left(x_{n}^{-}\right)$, where $z\left(x_{n}^{+}\right)$and $z\left(x_{n}^{-}\right)$are the right and left limits of $z$, respectively, at $x_{n}, n=1,2, \ldots, k$.

It is important to note that in this paper, we use the notations $\mathrm{HU}$ for Hyers-Ulam, GHU for generalized Hyers-Ulam, HUR for Hyers-Ulam-Rassias, and GHUR for generalized Hyers-Ulam-Rassis, respectively.

The rest of the paper is organized as follows. In Sect. 2, we give some definitions and preliminary results necessary in this study. In Sect. 3, we give results concerning the existence of solutions. In Sect. 4, we study the Hyers-Ulam stability and Hyers-Ulam-Rassias stability of problem (1). In Sect. 5, we illustrate the applications of our main results by providing a self-illustrative example. In Sect. 6, we conclude our work. 


\section{Preliminaries}

This section is concerned with introduction to some basic results and definitions.

Let $J=[0, T]$ with $0=x_{0}<x_{1}<x_{2}<\cdots<x_{n}=T$. We denote by $E$ the space $P C(J, R)$ of all piecewise continuous functions on $J$. More precisely, let $J=J_{0} \cup J_{1} \cup J_{2} \cup \cdots \cup J_{n}$, where $J_{0}=\left[x_{0}, x_{1}\right], J_{1}=\left(x_{1}, x_{2}\right], J_{2}=\left(x_{2}, x_{3}\right], \ldots, J_{n}=\left(x_{n}, x_{n+1}\right], n=1,2,3, \ldots, k$, and $J^{\prime}=J \backslash$ $\left\{x_{1}, x_{2}, \ldots, x_{n}\right\}$. Then

$$
E=\left\{z: J \rightarrow R: z \in C\left(J_{n}, R\right), \text { and } z\left(x_{n}^{+}\right), z\left(x_{n}^{-}\right) \text {exist }\right\}
$$

and $\Delta z\left(x_{n}\right)=z\left(x_{n}^{+}\right)-z\left(x_{n}^{-}\right)$for $n=1,2, \ldots, k$. The space $\left(E,\|\cdot\|_{E}\right)$ is a Banach space with respect to the norm $\|z\|_{E}=\max \{|z(x)|: x \in J\}$.

Definition 1 ([32]) The Riemann fractional integral of a function $z \in L^{1}\left([0, T], R_{+}\right)$is defined as

$$
{ }_{0} I_{x}^{\alpha} z(x)=\frac{1}{\Gamma(\alpha)} \int_{0}^{x}(x-\tau)^{\alpha-1} z(\tau) d \tau,
$$

where $\alpha \in(0, \infty)$ is the order of integration, and the integral on the right-hand side is pointwise defined on $(0, \infty)$.

Definition 2 ([6]) The Caputo fractional-order derivative of a function $z:(0, \infty) \rightarrow R$ is defined as

$$
{ }_{0}^{c} D_{x}^{\alpha} z(x)=\frac{1}{\Gamma(n-\alpha)} \int_{0}^{x}(x-\tau)^{n-\alpha-1} z^{(n)}(\tau) d \tau,
$$

where $n=[\alpha]+1$, and $[\alpha]$ is the integral part of a real number $\alpha$.

Lemma 1 ([33]) For $\alpha>0$, the solution of FDE

$$
{ }_{0}^{c} D_{x}^{\alpha} z(x)=h(x)
$$

is given by

$$
z(x)={ }_{0} I_{x}^{\alpha} h(x)+\sum_{i=0}^{n-1} \frac{z^{(i)}(0)}{i !} x^{i}
$$

where $n=[\alpha]+1$.

Let $\varphi \in C\left(J, R_{+}\right)$be a nondecreasing function. We assume that for $s \in E, \epsilon>0$, and $\omega \geq 0$, the following inequalities hold:

$$
\begin{aligned}
& \left\{\begin{array}{l}
\left|{ }_{0}^{c} D_{x_{n}}^{\alpha} s(x)-f\left(x, s(x), s(m x),{ }_{0}^{c} D_{x_{n}}^{\alpha} s(x)\right)\right| \leq \epsilon, \quad x \in J_{n}, n=1,2, \ldots, k, \\
\left|\Delta s\left(x_{n}\right)-I_{n}\left(s\left(x_{n}\right)\right)\right| \leq \epsilon, \quad n=1,2, \ldots, k
\end{array}\right. \\
& \left\{\begin{array}{l}
\left|{ }_{0}^{c} D_{x_{n}}^{\alpha} s(x)-f\left(x, s(x), s(m x),{ }_{0}^{c} D_{x_{n}}^{\alpha} s(x)\right)\right| \leq \varphi(x), \quad x \in J_{n}, n=1,2, \ldots, k, \\
\left|\Delta s\left(x_{n}\right)-I_{n}\left(s\left(x_{n}\right)\right)\right| \leq \omega, \quad n=1,2, \ldots, k
\end{array}\right.
\end{aligned}
$$


and

$$
\left\{\begin{array}{l}
\left|{ }_{0}^{c} D_{x_{n}}^{\alpha} s(x)-f\left(x, s(x), s(m x),{ }_{0}^{c} D_{x_{n}}^{\alpha} s(x)\right)\right| \leq \epsilon \varphi(x), \quad x \in J_{n}, n=1,2, \ldots, k, \\
\left|\Delta s\left(x_{n}\right)-I_{n}\left(s\left(x_{n}\right)\right)\right| \leq \epsilon \omega, \quad n=1,2, \ldots, k .
\end{array}\right.
$$

Definition 3 ([34]) Problem (1) is HU stable if there exists a real number $C_{f}>0$ such that for any solution $s \in E$ of inequality (2), there are a unique solution $z \in E$ of (1) and $\epsilon>0$ such that

$$
|s(x)-z(x)| \leq C_{f} \epsilon, \quad x \in J .
$$

Definition 4 ([34]) Problem (1) is GHU stable if there exists a real function $\vartheta \in C\left(R_{+}, R_{+}\right)$ with $\vartheta(0)=0$ such that for any solution $s \in E$ of inequality (2), there exist a unique solution $z \in E$ of (1) and $\epsilon>0$ such that

$$
|s(x)-z(x)| \leq \vartheta(\epsilon), \quad x \in J
$$

Definition 5 ([34]) Problem (1) is HUR stable with respect to $(\omega, \varphi)$ if there exists a real number $C_{f}>0$ such that for any solution $s \in E$ of inequality (4), there is a unique solution $z \in E$ of (1) such that

$$
|s(x)-z(x)| \leq C_{f} \epsilon(\omega+\varphi(x)), \quad x \in J .
$$

Definition 6 ([34]) Problem (1) is GHUR stable with respect to $(\omega, \varphi)$ if there exists $C_{f} \in$ $R>0$ such that for any solution $s \in E$ of inequality (3), there is a unique solution $z \in E$ of (1) such that

$$
|s(x)-z(x)| \leq C_{f}(\omega+\varphi(x)), \quad x \in J
$$

Remark 1 A function $s \in E$ is a solution of (2) if there are a function $\theta \in E$ and a sequence $\theta_{n}$ (which depends on $s$ ) such that

(i) $|\theta(x)| \leq \epsilon,\left|\theta_{n}\right| \leq \epsilon, x \in J_{n}, n=1,2, \ldots, k$;

(ii) ${ }_{0}^{c} D_{x_{n}}^{\alpha} s(x)=f\left(x, s(x), s(m x),{ }_{0}^{c} D_{x_{n}}^{\alpha} s(x)\right)+\theta(x), x \in J_{n}, n=1,2, \ldots, k$;

(iii) $\Delta s\left(x_{n}\right)=I_{n}\left(s\left(x_{n}\right)\right)+\theta_{n}, x \in J_{n}, n=1,2, \ldots, k$.

Remark 2 A function $s \in E$ is a solution of (3) if there are a function $\theta \in E$ and a sequence $\theta_{n}$ (which depends on $s$ ) such that

(i) $|\theta(x)| \leq \varphi(x),\left|\theta_{n}\right| \leq \omega, x \in J_{n}, n=1,2, \ldots, k$;

(ii) ${ }_{0}^{c} D_{x_{n}}^{\alpha} s(x)=f\left(x, s(x), s(m x),{ }_{0}^{c} D_{x_{n}}^{\alpha} s(x)\right)+\theta(x), x \in J_{n}, n=1,2, \ldots, k$;

(iii) $\Delta s\left(x_{n}\right)=I_{n}\left(s\left(x_{n}\right)\right)+\theta_{n}, x \in J_{n}, n=1,2, \ldots, k$.

Remark 3 A function $s \in E$ is a solution of (4) if there are a function $\theta \in E$ and sequence $\theta_{n}$ (which depends on $s$ ) such that

(i) $|\theta(x)| \leq \epsilon \varphi(x),\left|\theta_{n}\right| \leq \epsilon \omega, x \in J_{n}, n=1,2, \ldots, k$;

(ii) ${ }_{0}^{c} D_{x_{n}}^{\alpha} s(x)=f\left(x, s(x), s(m x),{ }_{0}^{c} D_{x_{n}}^{\alpha} s(x)\right)+\theta(x), x \in J_{n}, n=1,2, \ldots, k$;

(iii) $\Delta s\left(x_{n}\right)=I_{n}\left(s\left(x_{n}\right)\right)+\theta_{n}, x \in J_{n}, n=1,2, \ldots, k$. 
Theorem 1 ([35]) Let $\mathscr{W}: E \rightarrow$ E be a completely continuous operator, where $E$ is a Banach space, and $\Omega=\{z \in E: z=\delta \mathscr{W} z, 0<\delta<1\}$ is a bounded set. Then $\mathscr{W}$ has at least one fixed point in $E$.

\section{Existence of solution: main results}

In this section, we derive conditions for the existence and uniqueness of a solution for problem (1).

Lemma 2 Let $\psi: J \rightarrow R$ be a continuous function, and let $0<\alpha \leq 1$. Then a function $z \in E$ is a solution of the impulsive problem

$$
\left\{\begin{array}{l}
{ }_{0}^{c} D_{x_{n}}^{\alpha} z(x)=\psi(x), \quad x \in J, x \neq x_{n} \text { for } n=1,2, \ldots, k, \\
z(0)=z_{0}, \\
\Delta z\left(x_{n}\right)=I_{n}\left(z\left(x_{n}\right)\right), \quad n=1,2, \ldots, k,
\end{array}\right.
$$

if and only if $z$ satisfies the integral equation

$$
z(x)=\left\{\begin{array}{l}
\frac{1}{\Gamma(\alpha)} \int_{0}^{x}(x-\tau)^{\alpha-1} \psi(\tau) d \tau+z_{0}, \quad x \in J_{0}, \\
\frac{1}{\Gamma(\alpha)} \int_{x_{n}}^{x}(x-\tau)^{\alpha-1} \psi(\tau) d \tau+\sum_{i=1}^{n}\left[\frac{1}{\Gamma(\alpha)} \int_{x_{i-1}}^{x_{i}}\left(x_{i}-\tau\right)^{\alpha-1} \psi(\tau) d \tau\right. \\
\left.\quad+I_{i}\left(z\left(x_{i}\right)\right)\right]+z_{0}, \quad x \in J_{n}, n=1,2, \ldots, k .
\end{array}\right.
$$

Proof Let $z$ be a solution of (5). Then applying Lemma 1, for each $x \in J_{0}$, we have

$$
z(x)-z(0)={ }_{0} I_{x}^{\alpha} \psi(x)
$$

which implies

$$
z(x)=\frac{1}{\Gamma(\alpha)} \int_{0}^{x}(x-\tau)^{\alpha-1} \psi(\tau) d \tau+z(0) .
$$

Using the initial condition $z(0)=z_{0}$, from (7) we have

$$
z(x)=\frac{1}{\Gamma(\alpha)} \int_{0}^{x}(x-\tau)^{\alpha-1} \psi(\tau) d \tau+z_{0}, \quad x \in J_{0} .
$$

Similarly, for $x \in J_{1}$, we have

$$
z(x)=\frac{1}{\Gamma(\alpha)} \int_{x_{1}}^{x}(x-\tau)^{\alpha-1} \psi(\tau) d \tau+z\left(x_{1}\right)
$$

and

$$
z\left(x_{1}^{-}\right)=\frac{1}{\Gamma(\alpha)} \int_{0}^{x}(x-\tau)^{\alpha-1} \psi(\tau) d \tau+z_{0}, \quad z\left(x_{1}^{+}\right)=z\left(x_{1}\right)
$$

From

$$
\Delta z\left(x_{1}\right)=z\left(x_{1}^{+}\right)-z\left(x_{1}^{-}\right)=I_{1}\left(z\left(x_{1}\right)\right)
$$


we get

$$
z\left(x_{1}\right)=\frac{1}{\Gamma(\alpha)} \int_{0}^{x}(x-\tau)^{\alpha-1} \psi(\tau) d \tau+z_{0}+I_{1}\left(z\left(x_{1}\right)\right) .
$$

Putting for $z\left(x_{1}\right)$, (9) implies

$$
\begin{aligned}
z(x)= & \frac{1}{\Gamma(\alpha)} \int_{x_{1}}^{x}(x-\tau)^{\alpha-1} \psi(\tau) d \tau+\frac{1}{\Gamma(\alpha)} \int_{0}^{x}(x-\tau)^{\alpha-1} \psi(\tau) d \tau \\
& +z_{0}+I_{1}\left(z\left(x_{1}\right)\right), \quad x \in J_{1} .
\end{aligned}
$$

Generalizing in this way, for $x \in J_{n}$, we have

$$
\begin{aligned}
z(x)= & \frac{1}{\Gamma(\alpha)} \int_{x_{n}}^{x}(x-\tau)^{\alpha-1} \psi(\tau) d \tau+\sum_{i=1}^{n}\left[\frac{1}{\Gamma(\alpha)} \int_{x_{i-1}}^{x_{i}}\left(x_{i}-\tau\right)^{\alpha-1} \psi(\tau) d \tau\right. \\
& \left.+I_{i}\left(z\left(x_{i}\right)\right)\right]+z_{0}, \quad n=1,2, \ldots, k
\end{aligned}
$$

Thus from (8) and (10) we get (6).

Now, conversely, let $z$ be a solution of (6). Then taking the $\alpha$ th-order derivative of (6) gives the differential equation in (5). This completes the proof.

Corollary 1 From Lemma 2 we get the following solution for our problem (1):

$$
z(x)=\left\{\begin{array}{l}
\frac{1}{\Gamma(\alpha)} \int_{0}^{x}(x-\tau)^{\alpha-1} f\left(x, z(x), z(m x),{ }_{0}^{c} D_{x_{n}}^{\alpha} z(x)\right) d \tau+z_{0}, \quad x \in J_{0}, \\
\frac{1}{\Gamma(\alpha)} \int_{x_{n}}^{x}(x-\tau)^{\alpha-1} f\left(x, z(x), z(m x),{ }_{0}^{c} D_{x_{n}}^{\alpha} z(x)\right) d \tau \\
\quad+\sum_{i=1}^{n}\left[\frac{1}{\Gamma(\alpha)} \int_{x_{i-1}}^{x_{i}}\left(x_{i}-\tau\right)^{\alpha-1} f\left(x, z(x), z(m x),{ }_{0}^{c} D_{x_{n}}^{\alpha} z(x)\right) d \tau\right. \\
\left.\quad+I_{i}\left(z\left(x_{i}\right)\right)\right]+z_{0}, \quad x \in J_{n}, n=1,2, \ldots, k .
\end{array}\right.
$$

We use the notation $u_{z}(x)=f\left(x, z(x), z(m x),{ }_{0}^{c} D_{x_{n}}^{\alpha} z(x)\right)=f\left(x, z(x), z(m x), u_{z}(x)\right)$.

The following assumptions are necessary in obtaining the main results.

$\left(A_{1}\right) f: J \times R^{3} \rightarrow R$ is continuous;

$\left(A_{2}\right)$ There exist constants $M_{f}>0$ and $0<N_{f}<1$ such that for all $x \in J$ and $z, \bar{z} \in R$, we have the following relation:

$$
\begin{aligned}
& \left|f\left(x, z(x), z(m x), u_{z}(x)\right)-f\left(x, \bar{z}(x), \bar{z}(m x), u_{\bar{z}}(x)\right)\right| \\
& \leq M_{f}(|z(x)-\bar{z}(x)|+|z(m x)-\bar{z}(m x)|) \\
& \quad+N_{f}\left|u_{z}(x)-u_{\bar{z}}(x)\right| .
\end{aligned}
$$

$\left(A_{3}\right)$ For any $z, \bar{z} \in E$, there exists a constant $A_{I}^{*}>0$ such that

$$
\left|I_{i}\left(z\left(x_{i}\right)\right)-I_{i}\left(\bar{z}\left(x_{i}\right)\right)\right| \leq A_{I}^{*}\left|z\left(x_{i}\right)-\bar{z}\left(x_{i}\right)\right|
$$

$\left(A_{4}\right)$ there exist functions $a, b, c \in C\left(J, R^{+}\right)$such that

$$
\left|f\left(x, z(x), z(m x), u_{z}(x)\right)\right| \leq a(x)+b(x)(|z(x)|+|z(m x)|)+c(x)\left|u_{z}\right|
$$

with $c^{*}=\sup _{x \in J} c(x)<1$; 
$\left(A_{5}\right)$ Let for each $z \in R, i=1,2, \ldots, n$, there exist constants $K, L>0$, such that $\left|I_{i}(z)\right| \leq$ $K|z|+L$

$\left(A_{6}\right)$ Let for a nondecreasing function $\varphi \in P C\left(J, R_{+}\right)$and constant $\varrho_{\varphi}>0$, the following inequality hold:

$$
I^{\alpha} \varphi(x) \leq \varrho_{\varphi} \varphi(x), \quad x \in J
$$

We convert our problem to a fixed-point problem by defining the operator $\mathscr{W}: C(J, R) \rightarrow$ $C(J, R)$ as

$$
\left\{\begin{aligned}
(\mathscr{W} z)(x)= & \frac{1}{\Gamma(\alpha)} \int_{0}^{x}(x-\tau)^{\alpha-1} u_{z}(\tau) d \tau+z_{0}, \quad x \in J_{0}, \\
(\mathscr{W} z)(x)= & \frac{1}{\Gamma(\alpha)} \int_{x_{n}}^{x}(x-\tau)^{\alpha-1} u_{z}(\tau) d \tau+\sum_{i=1}^{n}\left[\frac{1}{\Gamma(\alpha)} \int_{x_{i-1}}^{x_{i}}\left(x_{i}-\tau\right)^{\alpha-1} u_{z}(\tau) d \tau\right. \\
& \left.+I_{i}\left(z\left(x_{i}\right)\right)\right]+z_{0}, \quad n=1,2, \ldots, k
\end{aligned}\right.
$$

Theorem 2 Suppose conditions $\left(A_{1}\right)-\left(A_{5}\right)$ are satisfied. Then problem (1) has at least one solution in the given interval.

Proof The proof is divided into four steps.

Step 1: $\mathscr{W}$ is continuous. Let $\left\{z_{n}\right\} \in C(J, R)$ be a sequence such that $z_{n} \rightarrow z \in C(J, R)$. For each $x \in J_{n}$, we have

$$
\begin{aligned}
& \left|\left(\mathscr{W} z_{n}\right)(x)-(\mathscr{W} z)(x)\right| \\
& \leq \frac{1}{\Gamma(\alpha)} \int_{x_{n}}^{x}(x-\tau)^{\alpha-1}\left|u_{z_{n}}(\tau)-u_{z}(\tau)\right| d \tau \\
& \quad+\sum_{i=1}^{n} \frac{1}{\Gamma(\alpha)} \int_{x_{i-1}}^{x_{i}}\left(x_{i}-\tau\right)^{\alpha-1}\left|u_{z_{n}}(\tau)-u_{z}(\tau)\right| d \tau+\sum_{i=1}^{n}\left|I_{i}\left(z_{n}\left(x_{i}\right)\right)-I_{i}\left(z\left(x_{i}\right)\right)\right|,
\end{aligned}
$$

where $u_{z_{n}}, u_{z} \in C(J, R)$ satisfy

$$
\begin{aligned}
& u_{z_{n}}(x)=f\left(x, z_{n}(x), z_{n}(m x), u_{z_{n}}(x)\right), \\
& u_{z}(x)=f\left(x, z(x), z(m x), u_{z}(x)\right) .
\end{aligned}
$$

By $\left(A_{2}\right)$ we get

$$
\left|u_{z_{n}}(x)-u_{z}(x)\right| \leq \frac{2 M_{f}}{1-N_{f}}\left|z_{n}(x)-z(x)\right| .
$$

Now $z_{n} \rightarrow z$ as $n \rightarrow \infty$ implies $u_{z_{n}}(x) \rightarrow u_{z}(x)$ for each $x \in J_{n}$. We know that every convergent sequence is bounded. So let $\aleph>0$ be such that for each $x \in J$, we have $\left|u_{z_{n}}(x)\right| \leq \aleph$ and $\left|u_{z}(x)\right| \leq \aleph$. Then

$$
\begin{aligned}
(x-\tau)^{\alpha-1}\left|u_{z_{n}}(\tau)-u_{z}(\tau)\right| & \leq(x-\tau)^{\alpha-1}\left(\left|u_{z_{n}}(\tau)\right|+\left|u_{z}(\tau)\right|\right) \\
& \leq 2 \aleph(x-\tau)^{\alpha-1}, \\
\left(x_{i}-\tau\right)^{\alpha-1}\left|u_{z_{n}}(\tau)-u_{z}(\tau)\right| & \leq\left(x_{i}-\tau\right)^{\alpha-1}\left(\left|u_{z_{n}}(\tau)\right|+\left|u_{z}(\tau)\right|\right)
\end{aligned}
$$




$$
\leq 2 \aleph\left(x_{i}-\tau\right)^{\alpha-1} .
$$

For each $x \in J_{n}$, the functions $\tau \rightarrow 2 \aleph(x-\tau)^{\alpha-1}$ and $\tau \rightarrow 2 \aleph\left(x_{i}-\tau\right)^{\alpha-1}$ are integrable. Also, $f$ and $I$ are continuous. Thus applying the Lebesgue dominated convergence theorem, we have $\left|\left(\mathscr{W} z_{n}\right)(x)-(\mathscr{W} z)(x)\right| \rightarrow 0$ as $n \rightarrow \infty$. Hence, in particular, $\max _{x \in J} \mid \mathscr{W} z_{n}(x)-$ $\mathscr{W} z(x) \mid \rightarrow 0$ as $n \rightarrow \infty$, which implies that $\left\|\mathscr{W} z_{n}-\mathscr{W} z\right\|_{E} \rightarrow 0$ as $n \rightarrow \infty$. Similarly, for each $x \in J_{0}$, we can show that $\left\|\mathscr{W} z_{n}-\mathscr{W} z\right\|_{E} \rightarrow 0$ as $n \rightarrow \infty$. Therefore $\mathscr{W}$ is continuous.

Step 2: Here we show that $\mathscr{W}$ is bounded. Let for $\gamma>0$, there exist a positive real number $\Theta>0$ such that for each $z \in \Omega_{\gamma}=\left\{z \in E:\|z\|_{E} \leq \gamma\right\}$, we have $\|\mathscr{W}(z)\|_{E} \leq \Theta$.

For $x \in J_{n}$, consider

$$
\begin{aligned}
|(\mathscr{W} z)(x)| \leq & \frac{1}{\Gamma(\alpha)} \int_{x_{n}}^{x}(x-\tau)^{\alpha-1}\left|u_{z}(\tau)\right| d \tau+\sum_{i=1}^{n} \frac{1}{\Gamma(\alpha)} \int_{x_{i-1}}^{x_{i}}\left(x_{i}-\tau\right)^{\alpha-1}\left|u_{z}(\tau)\right| d \tau \\
& +\sum_{i=1}^{n}\left|I_{i}\left(z\left(x_{i}\right)\right)\right|+\left|z_{0}\right| .
\end{aligned}
$$

By $\left(A_{4}\right)$ for $x \in J_{n}$, we have

$$
\begin{aligned}
\left|u_{z}(x)\right| & =\left|f\left(x, z(x), z(m x), u_{z}(x)\right)\right| \\
& \leq a(x)+b(x)(|z(x)|+|z(m x)|)+c(x)\left|u_{z}(x)\right| \\
& \leq a(x)+2 b(x)\|z\|_{E}+c(x)\left|u_{z}(x)\right| \\
& \leq a(x)+2 b(x) \gamma+c(x)\left|u_{z}(x)\right| \\
& \leq a^{*}+2 b^{*} \gamma+c^{*}\left|u_{z}(x)\right|,
\end{aligned}
$$

where $a^{*}=\sup _{x \in J_{n}} a(x), b^{*}=\sup _{x \in J_{n}} b(x)$, and $c^{*}=\sup _{x \in J_{n}} c(x)<1$.

Then

$$
\left|u_{z}(x)\right| \leq \frac{a^{*}+2 b^{*} \gamma}{1-c^{*}}=\mu .
$$

By application of assumptions $\left(A_{4}\right)-\left(A_{5}\right)$ we obtain

$$
\begin{aligned}
|(\mathscr{W} z)(x)| & \leq \frac{\mu}{\Gamma(\alpha)} \int_{x_{n}}^{x}(x-\tau)^{\alpha-1} d \tau+\frac{\mu}{\Gamma(\alpha)} \sum_{i=1}^{n} \int_{x_{i-1}}^{x_{i}}\left(x_{i}-\tau\right)^{\alpha-1} d \tau+n(K|z|+L)+\zeta \\
& \leq \frac{\mu(1+n) T^{\alpha}}{\Gamma(\alpha+1)}+n(K \gamma+L)+\zeta=\Theta .
\end{aligned}
$$

Thus

$$
\|\mathscr{W} z\|_{E} \leq \frac{\mu(1+n) T^{\alpha}}{\Gamma(\alpha+1)}+n(K \gamma+L)+\zeta=\Theta
$$

Similarly, for $x \in J_{0}$, we can show that

$$
\|\mathscr{W} z\|_{E} \leq \Theta .
$$


Step 3: We show $\mathscr{W}$ maps bounded sets into equicontinuous sets of $E$.

Let $x_{1}, x_{2} \in J_{n}$ be such that $x_{1}<x_{2}$. As in Step 2 , take a bounded set $\Omega_{\gamma^{*}}$. For $z \in \Omega_{\gamma^{*}}$, we have

$$
\begin{aligned}
& \left|(\mathscr{W} z)\left(x_{2}\right)-(\mathscr{W} z)\left(x_{1}\right)\right| \\
& \leq\left|\frac{1}{\Gamma(\alpha)} \int_{x_{n}}^{x_{2}}\left(x_{2}-\tau\right)^{\alpha-1} u_{z}(\tau) d \tau-\frac{1}{\Gamma(\alpha)} \int_{x_{n}}^{x_{1}}\left(x_{1}-\tau\right)^{\alpha-1} u_{z}(\tau)\right| d \tau \\
& \quad+\sum_{0<x_{n}<x_{2}-x_{1}} \frac{1}{\Gamma(\alpha)} \int_{x_{n-1}}^{x_{n}}\left(x_{n}-\tau\right)^{\alpha-1}\left|u_{z}(\tau)\right| d \tau+\sum_{0<x_{n}<x_{2}-x_{1}}\left|I_{n}\left(z\left(x_{n}\right)\right)\right| .
\end{aligned}
$$

We see that the right-hand side of (13) approaches 0 as $x_{1} \rightarrow x_{2}$. Therefore $\mid(\mathscr{W} z)\left(x_{2}\right)-$ $(\mathscr{W} z)\left(x_{1}\right) \mid \rightarrow 0$ as $x_{1} \rightarrow x_{2}$. Similarly, in a subinterval $J_{0}$, we can show that $\mid(\mathscr{W} z)\left(x_{2}\right)-$ $(\mathscr{W} z)\left(x_{1}\right) \mid \rightarrow 0$ as $x_{1} \rightarrow x_{2}$. Therefore, as a result of Steps 1-3 and the Ascoli-Arzelà theorem, we conclude that $\mathscr{W}: E \rightarrow E$ is completely continuous.

Step 4: In this final step, we define the set $\mho=\{z \in E: z=\delta \mathscr{W} z$ for some $0<\delta<1\}$. We need to show that $\mho$ is bounded. Let $z \in \mho$. Then $z=\delta \mathscr{W} z$ for some $0<\delta<1$. Hence for each $x \in J_{n}$, we have

$$
\begin{aligned}
z(x)= & \frac{\delta}{\Gamma(\alpha)} \int_{x_{n}}^{x}(x-\tau)^{\alpha-1} u_{z}(\tau) d \tau+\delta \sum_{0<x_{n}<x} \frac{1}{\Gamma(\alpha)} \int_{x_{n-1}}^{x_{n}}\left(x_{n}-\tau\right)^{\alpha-1} u_{z}(\tau) d \tau \\
& +\delta \sum_{0<x_{n}<x} I_{n}\left(z\left(x_{n}\right)\right)+\delta z_{0} .
\end{aligned}
$$

Now since $0<\delta<1$, from the above we have

$$
\begin{aligned}
|z(x)| \leq & \frac{a^{*}+2 b^{*}\|z\|_{E}}{\left(1-c^{*}\right) \Gamma(\alpha)} \int_{x_{n}}^{x}(x-\tau)^{\alpha-1} d \tau+\sum_{0<x_{n}<x} \frac{a^{*}+2 b^{*}\|z\|_{E}}{\left(1-c^{*}\right) \Gamma(\alpha)} \int_{x_{n-1}}^{x_{n}}\left(x_{n}-\tau\right)^{\alpha-1} d \tau \\
& +\sum_{0<x_{n}<x}\left|I_{n}\left(z\left(x_{n}\right)\right)\right|+\left|z_{0}\right|,
\end{aligned}
$$

from which we have

$$
\|z\|_{E} \leq \frac{a^{*}+2 b^{*}\|z\|_{E} T^{\alpha}}{\left(1-c^{*}\right) \Gamma(\alpha+1)}+\frac{n\left(a^{*}+2 b^{*}\|z\|_{E}\right) T^{\alpha}}{\left(1-c^{*}\right) \Gamma(\alpha+1)}+n(K \gamma+L)+\zeta=: \mathbf{K} .
$$

A similar result can be achieved for $x \in J_{0}$. Therefore $\mho$ is a bounded set. Hence, by applying Schaefer's fixed point theorem, $\mathscr{W}$ has at leat one confirmed fixed point.

Theorem 3 Assume that conditions $\left(A_{1}\right)-\left(A_{3}\right)$ together with the inequality

$$
\left(\frac{2 M_{f} T^{\alpha}}{\left(1-N_{f}\right) \Gamma(\alpha+1)}+\frac{2 M_{f} n T^{\alpha}}{\left(1-N_{f}\right) \Gamma(\alpha+1)}+A_{I}^{*} n\right)<1
$$

are satisfied. Then (1) has a unique solution.

Proof For $z, \bar{z} \in E$ and $x \in J_{n}$, we have

$$
|(\mathscr{W} z)(x)-(\mathscr{W} \bar{z})(x)| \leq \frac{1}{\Gamma(\alpha)} \int_{x_{n}}^{x}(x-\tau)^{\alpha-1}\left|u_{z}(\tau)-w_{\bar{z}}(\tau)\right| d \tau
$$




$$
\begin{aligned}
& +\sum_{i=1}^{n} \frac{1}{\Gamma(\alpha)} \int_{x_{i}-1}^{x_{i}}\left(x_{i}-\tau\right)^{\alpha-1}\left|u_{z}(\tau)-w_{\bar{z}}(\tau)\right| d \tau \\
& +\sum_{i=1}^{n}\left|I\left(z\left(x_{i}\right)\right)-I\left(\bar{z}\left(x_{i}\right)\right)\right|
\end{aligned}
$$

where $u_{z}, w_{z} \in C(J, R)$ are given by

$$
\begin{aligned}
& u_{z}(x)=f\left(x, z(x), z(m x), u_{z}(x)\right), \\
& w_{\bar{z}}(x)=f\left(x, \bar{z}(x), \bar{z}(m x), w_{\bar{z}}(x)\right) .
\end{aligned}
$$

By $\left(A_{2}\right)$ we have

$$
\begin{aligned}
\left|u_{z}(x)-w_{\bar{z}}(x)\right| & =\left|f\left(x, z(x), z(m x), u_{z}(x)\right)-f\left(x, \bar{z}(x), \bar{z}(m x), w_{\bar{z}}(x)\right)\right| \\
& \leq M_{f}(|z(x)-\bar{z}(x)|+|z(m x)-\bar{m} z(x)|)+N_{f}\left|u_{z}(x)-w_{\bar{z}}(x)\right| \\
& \leq 2 M_{f}\left(|z(x)-\bar{z}(x)|+N_{f}\left|u_{z}(x)-w_{\bar{z}}(x)\right| .\right.
\end{aligned}
$$

Then

$$
\left|u_{z}(x)-w_{\bar{z}}(x)\right| \leq \frac{2 M_{f}}{1-N_{f}}|z(x)-\bar{z}(x)| .
$$

Thus by assumptions $\left(A_{2}\right)-\left(A_{3}\right)$ inequality (15) implies

$$
\begin{aligned}
&|(\mathscr{W} z)(x)-(\mathscr{W} \bar{z})(x)| \\
& \leq \frac{2 M_{f}}{\left(1-N_{f}\right) \Gamma(\alpha)} \int_{x_{n}}^{x}(x-\tau)^{\alpha-1}|z(\tau)-\bar{z}(\tau)| d \tau \\
& \quad+\sum_{i=1}^{n} \frac{2 M_{f}}{\left(1-N_{f}\right) \Gamma(\alpha)} \int_{x_{i}-1}^{x_{i}}\left(x_{i}-\tau\right)^{\alpha-1}|z(\tau)-\bar{z}(\tau)| d \tau \\
& \quad+\sum_{i=1}^{n} A_{I}^{*}|z(x)-\bar{z}(x)| \\
& \leq\left(\frac{2 M_{f} T^{\alpha}}{\left(1-N_{f}\right) \Gamma(\alpha+1)}+\frac{2 M_{f} n T^{\alpha}}{\left(1-N_{f}\right) \Gamma(\alpha+1)}+A_{I}^{*} n\right)|z(x)-\bar{z}(x)| .
\end{aligned}
$$

Taking the maximum norm, we get

$$
\|\mathscr{W} z-\mathscr{W} \bar{z}\|_{E} \leq\left(\frac{2 M_{f} T^{\alpha}}{\left(1-N_{f}\right) \Gamma(\alpha+1)}+\frac{2 M_{f} n T^{\alpha}}{\left(1-N_{f}\right) \Gamma(\alpha+1)}+A_{I}^{*} n\right)\|z-\bar{z}\|_{E} .
$$

Similarly, for $z, \bar{z} \in E$ and $x \in J_{0}$, we get

$$
\|\mathscr{W} z-\mathscr{W} \bar{z}\|_{E} \leq\left(\frac{2 M_{f} T^{\alpha}}{\left(1-N_{f}\right) \Gamma(\alpha+1)}\right)\|z-\bar{z}\|_{E}
$$

Since

$$
\left(\frac{2 M_{f} T^{\alpha}}{\left(1-N_{f}\right) \Gamma(\alpha+1)}\right) \leq\left(\frac{2 M_{f} T^{\alpha}}{\left(1-N_{f}\right) \Gamma(\alpha+1)}+\frac{2 M_{f} n T^{\alpha}}{\left(1-N_{f}\right) \Gamma(\alpha+1)}+A_{I}^{*} n\right)<1,
$$


$\mathscr{W}$ is contraction operator, and therefore by Banach's contraction principle $\mathscr{W}$ has a unique fixed point in the given interval $J$. Thus problem (1) has a unique solution.

\section{Hyers-Ulam-type stability analysis}

Here we study the Hyers-Ulam stability of problem (1).

Theorem 4 Assume that conditions $\left(A_{1}\right)-\left(A_{5}\right)$ and (14) hold. Then proposed problem (1) is HU stable.

Proof Let $s \in E$ be any solution of (2), and let $z$ be the unique solution of

$$
\left\{\begin{array}{l}
{ }_{0}^{c} D_{x_{n}}^{\alpha} z(x)=f\left(x, z(x), z(m x),{ }_{0}^{c} D_{x_{n}}^{\alpha} z(x)\right), \quad x \in J, \\
x \neq x_{n} \quad \text { for } n=1,2, \ldots, k, 0<\alpha \leq 1, \\
z(0)=z_{0} \\
\Delta z\left(x_{n}\right)=I_{n}\left(z\left(x_{n}\right)\right), \quad n=1,2, \ldots, k
\end{array}\right.
$$

By Lemma 2, for $x \in J_{n}$, we have

$$
\begin{aligned}
z(x)= & \frac{1}{\Gamma(\alpha)} \int_{x_{n}}^{x}(x-\tau)^{\alpha-1} u_{z}(\tau) d \tau+\sum_{i=1}^{n}\left[\frac{1}{\Gamma(\alpha)} \int_{x_{i-1}}^{x_{i}}\left(x_{i}-\tau\right)^{\alpha-1} u_{z}(\tau) d \tau\right. \\
& \left.+I_{i}\left(z\left(x_{i}\right)\right)\right]+z_{0}, x \in J_{n}, \quad n=1,2, \ldots, k
\end{aligned}
$$

By Remark 1 we get

$$
\left\{\begin{array}{l}
{ }_{0}^{c} D_{x_{n}}^{\alpha} s(x)=f\left(x, s(x), s(m x),{ }_{0}^{c} D_{x_{n}}^{\alpha} s(x)\right)+\theta(x), \quad x \in J \\
x \neq x_{n} \quad \text { for } n=1,2, \ldots, k, 0<\alpha \leq 1 \\
s(0)=s_{0} \\
\Delta s\left(x_{n}\right)=I_{n}\left(s\left(x_{n}\right)\right)+\theta_{n}, \quad n=1,2, \ldots, k
\end{array}\right.
$$

The solution of (18) is

$$
s(x)=\left\{\begin{array}{l}
\frac{1}{\Gamma(\alpha)} \int_{0}^{x}(x-\tau)^{\alpha-1} \bar{u}_{z}(\tau) d \tau+\frac{1}{\Gamma(\alpha)} \int_{0}^{x}(x-\tau)^{\alpha-1} \theta(\tau) d \tau+s_{0}, \quad x \in J_{0}, \\
\frac{1}{\Gamma(\alpha)} \int_{x_{n}}^{x}(x-\tau)^{\alpha-1} \bar{u}_{z}(\tau) d \tau+\frac{1}{\Gamma(\alpha)} \int_{x_{n}}^{x}(x-\tau)^{\alpha-1} \theta(\tau) d \tau \\
\quad+\sum_{i=1}^{n} \frac{1}{\Gamma(\alpha)} \int_{x_{i-1}}^{x_{i}}\left(x_{i}-\tau\right)^{\alpha-1} \bar{u}_{z}(\tau) d \tau+\sum_{i=1}^{n} \frac{1}{\Gamma(\alpha)} \int_{x_{i-1}}^{x_{i}}\left(x_{i}-\tau\right)^{\alpha-1} \theta(\tau) d \tau \\
\quad+\sum_{i=1}^{n} I_{i}\left(s\left(x_{i}\right)\right)+\sum_{i=1}^{n} \theta_{i}+s_{0}, \quad x \in J_{n}, n=1,2, \ldots, k,
\end{array}\right.
$$

where $\bar{u}_{z} \in C(J, R)$ is given by

$$
\bar{u}_{z}(x)=f\left(x, s(x), \bar{u}_{z}(x) .\right.
$$

Therefore, for each $x \in J_{n}$, we have

$$
|s(x)-z(x)|
$$




$$
\begin{aligned}
\leq & \frac{1}{\Gamma(\alpha)} \int_{x_{n}}^{x}(x-\tau)^{\alpha-1}\left|\bar{u}_{z}(\tau)-u_{z}(\tau)\right| d \tau+\frac{1}{\Gamma(\alpha)} \int_{x_{n}}^{x}(x-\tau)^{\alpha-1}|\theta(\tau)| d \tau \\
& +\sum_{i=1}^{n} \frac{1}{\Gamma(\alpha)} \int_{x_{i-1}}^{x_{i}}\left(x_{i}-\tau\right)^{\alpha-1}\left|\bar{u}_{z}(\tau)-u_{z}(\tau)\right| d \tau+\sum_{i=1}^{n} \frac{1}{\Gamma(\alpha)} \int_{x_{i-1}}^{x_{i}}\left(x_{i}-\tau\right)^{\alpha-1}|\theta(\tau)| d \tau \\
& +\sum_{i=1}^{n}\left|I_{i}\left(s\left(x_{i}\right)\right)-I_{i}\left(z\left(x_{i}\right)\right)\right|+\sum_{i=1}^{n}\left|\theta_{i}\right|, \quad x \in J_{n}, n=1,2, \ldots, k .
\end{aligned}
$$

By $\left(A_{2}\right)$ we obtain

$$
\left|\bar{u}_{z}(x)-u_{z}(x)\right| \leq \frac{2 M_{f}}{1-N_{f}}|s(x)-z(x)| .
$$

Hence applying assumptions $\left(A_{1}\right)-\left(A_{4}\right)$ and Remark 1 , we obtain

$$
\begin{aligned}
\mid s(x) & -z(x) \mid \\
\leq & \frac{2 M_{f}}{\left(1-N_{f}\right) \Gamma(\alpha)} \int_{x_{n}}^{x}(x-\tau)^{\alpha-1}|s(\tau)-z(\tau)| d \tau+\frac{\epsilon}{\Gamma(\alpha)} \int_{x_{n}}^{x}(x-\tau)^{\alpha-1} d \tau \\
& +\frac{2 M_{f}}{\left(1-N_{f}\right) \Gamma(\alpha)} \sum_{i=1}^{n} \int_{x_{i-1}}^{x_{i}}\left(x_{i}-\tau\right)^{\alpha-1}|s(\tau)-z(\tau)| d \tau+\sum_{i=1}^{n} \frac{\epsilon}{\Gamma(\alpha)} \int_{x_{i-1}}^{x_{i}}\left(x_{i}-\tau\right)^{\alpha-1} d \tau \\
& +\sum_{i=1}^{n} A_{I}^{*}\left|s\left(x_{i}\right)-z\left(x_{i}\right)\right|+\sum_{i=1}^{n} \epsilon, \quad x \in J_{n}, n=1,2, \ldots, k .
\end{aligned}
$$

By taking the maximum norm and simplification we get

$$
\begin{aligned}
\| s & -z \|_{E} \\
& \leq \epsilon\left(\frac{(1+n) T^{\alpha}}{\Gamma(\alpha+1)}+n\right)+\left(\frac{2 M_{f} T^{\alpha}}{\left(1-N_{f}\right) \Gamma(\alpha+1)}+\frac{2 M_{f} n T^{\alpha}}{\left(1-N_{f}\right) \Gamma(\alpha+1)}+A_{I}^{*} n\right)\|s-z\|_{E},
\end{aligned}
$$

from which we obtain

$$
\|s-z\|_{E} \leq \frac{\epsilon\left(\frac{(1+n) T^{\alpha}}{\Gamma(\alpha+1)}+n\right)}{1-\left(\frac{2 M_{f} T^{\alpha}}{\left(1-N_{f}\right) \Gamma(\alpha+1)}+\frac{2 M_{f} n T^{\alpha}}{\left(1-N_{f}\right) \Gamma(\alpha+1)}+A_{I}^{*} n\right)} .
$$

Similarly, for $x \in J_{0}$, we have

$$
\|s-z\|_{E} \leq \frac{\epsilon\left(\frac{T^{\alpha}}{\Gamma(\alpha+1)}\right)}{1-\left(\frac{2 M_{f} T^{\alpha}}{\left(1-N_{f}\right) \Gamma(\alpha+1)}\right)} .
$$

Using (19) and (20), for $x \in J$, we have

$$
\|s-z\|_{E} \leq \epsilon\left[\frac{\left(\frac{(1+n) T^{\alpha}}{\Gamma(\alpha+1)}+n\right)}{1-\left(\frac{2 M_{f} T^{\alpha}}{\left(1-N_{f}\right) \Gamma(\alpha+1)}+\frac{2 M_{f} n T^{\alpha}}{\left(1-N_{f}\right) \Gamma(\alpha+1)}+A_{I}^{*} n\right)}+\frac{\frac{T^{\alpha}}{\Gamma(\alpha+1)}}{1-\left(\frac{2 M_{f} T^{\alpha}}{\left(1-N_{f}\right) \Gamma(\alpha+1)}\right)}\right] .
$$

Thus

$$
\|s-z\|_{E} \leq C_{1} \epsilon
$$


where

$$
C_{1}=\left[\frac{\left(\frac{(1+n) T^{\alpha}}{\Gamma(\alpha+1)}+n\right)}{1-\left(\frac{2 M_{f} T^{\alpha}}{\left(1-N_{f}\right) \Gamma(\alpha+1)}+\frac{2 M_{f} n T^{\alpha}}{\left(1-N_{f}\right) \Gamma(\alpha+1)}+A_{I}^{*} n\right)}+\frac{\frac{T^{\alpha}}{\Gamma(\alpha+1)}}{1-\left(\frac{2 M_{f} T^{\alpha}}{\left(1-N_{f}\right) \Gamma(\alpha+1)}\right)}\right] .
$$

Therefore problem (1) is HU stable.

Corollary 2 If we set $\vartheta(\epsilon)=C_{f}(\epsilon)$ with $\vartheta(0)=0$, then problem (1) becomes GHU stable.

Theorem 5 If assumptions $\left(A_{1}\right)-\left(A_{8}\right)$ and (14) hold, then problem (1) is HUR stable with respect to $(\omega, \varphi)$.

Proof Let $s \in E$ be any solution of (4), and let $z$ be a unique solution of

$$
\left\{\begin{array}{l}
{ }_{0}^{c} D_{x_{n}}^{\alpha} z(x)=f\left(x, z(x), z(m x),{ }_{0}^{c} D_{x_{n}}^{\alpha} z(x)\right), \quad x \in J, x \neq x_{n} \text { for } n=1,2, \ldots, k, 0<\alpha \leq 1, \\
z(0)=z_{0}, \\
\Delta z\left(x_{n}\right)=I_{n}\left(z\left(x_{n}\right)\right), \quad n=1,2, \ldots, k .
\end{array}\right.
$$

Then from the proof of Theorem 4, for each $x \in J_{n}$, we have

$$
\begin{aligned}
\mid s(x) & -z(x) \mid \\
\leq & \frac{1}{\Gamma(\alpha)} \int_{x_{n}}^{x}(x-\tau)^{\alpha-1}\left|\bar{u}_{z}(\tau)-u_{z}(\tau)\right| d \tau+\frac{1}{\Gamma(\alpha)} \int_{x_{n}}^{x}(x-\tau)^{\alpha-1}|\theta(\tau)| d \tau \\
& +\sum_{i=1}^{n} \frac{1}{\Gamma(\alpha)} \int_{x_{i-1}}^{x_{i}}\left(x_{i}-\tau\right)^{\alpha-1}\left|\bar{u}_{z}(\tau)-u_{z}(\tau)\right| d \tau+\sum_{i=1}^{n} \frac{1}{\Gamma(\alpha)} \int_{x_{i-1}}^{x_{i}}\left(x_{i}-\tau\right)^{\alpha-1}|\theta(\tau)| d \tau \\
& +\sum_{i=1}^{n}\left|I_{i}\left(s\left(x_{i}\right)\right)-I_{i}\left(z\left(x_{i}\right)\right)\right|+\sum_{i=1}^{n}\left|\theta_{i}\right|, \quad x \in J_{n}, n=1,2, \ldots, k .
\end{aligned}
$$

By $\left(A_{2}\right)$ we obtain

$$
\left|\bar{u}_{z}(x)-u_{z}(x)\right| \leq \frac{2 M_{f}}{1-N_{f}}|s(x)-z(x)| .
$$

By assumptions $\left(A_{1}\right)-\left(A_{4}\right)$ and Remark 3 we have

$$
\begin{aligned}
\mid s(x) & -z(x) \mid \\
\leq & \frac{2 M_{f}}{\left(1-N_{f}\right) \Gamma(\alpha)} \int_{x_{n}}^{x}(x-\tau)^{\alpha-1}|s(\tau)-z(\tau)| d \tau+\frac{\epsilon}{\Gamma(\alpha)} \int_{x_{n}}^{x}(x-\tau)^{\alpha-1} \varphi(\tau) d \tau \\
& +\frac{2 M_{f}}{\left(1-N_{f}\right) \Gamma(\alpha)} \sum_{i=1}^{n} \int_{x_{i-1}}^{x_{i}}\left(x_{i}-\tau\right)^{\alpha-1}|s(\tau)-z(\tau)| d \tau \\
& +\sum_{i=1}^{n} \frac{\epsilon}{\Gamma(\alpha)} \int_{x_{i-1}}^{x_{i}}\left(x_{i}-\tau\right)^{\alpha-1} \varphi(\tau) d \tau \\
& +\sum_{i=1}^{n} A_{I}^{*}\left|s\left(x_{i}\right)-z\left(x_{i}\right)\right|+\epsilon \sum_{i=1}^{n} \omega, \quad x \in J_{n}, n=1,2, \ldots, k .
\end{aligned}
$$


Using $\left(A_{8}\right)$ and taking the maximum norm, we get

$$
\begin{aligned}
\|s-z\|_{E} \leq & \epsilon\left(\varrho_{\varphi} \varphi(x)(1+k)+k \omega\right) \\
& +\left(\frac{2 M_{f} T^{\alpha}}{\left(1-N_{f}\right) \Gamma(\alpha+1)}+\frac{2 M_{f} n T^{\alpha}}{\left(1-N_{f}\right) \Gamma(\alpha+1)}+A_{I}^{*} n\right)\|s-z\|_{E} \\
\leq & \epsilon(\varphi(x)+\omega)\left(\varrho_{\varphi}(1+k)+k\right) \\
& +\left(\frac{2 M_{f} T^{\alpha}}{\left(1-N_{f}\right) \Gamma(\alpha+1)}+\frac{2 M_{f} n T^{\alpha}}{\left(1-N_{f}\right) \Gamma(\alpha+1)}+A_{I}^{*} n\right)\|s-z\|_{E},
\end{aligned}
$$

which yields

$$
\|s-z\|_{E} \leq \frac{\epsilon(\varphi(x)+\omega)\left(\varrho_{\varphi}(1+k)+k\right)}{1-\left(\frac{2 M_{f} T^{\alpha}}{\left(1-N_{f}\right) \Gamma(\alpha+1)}+\frac{2 M_{f} n T^{\alpha}}{\left(1-N_{f}\right) \Gamma(\alpha+1)}+A_{I}^{*} n\right)} .
$$

Similarly, for $x \in J_{0}$, we have

$$
\|s-z\|_{E} \leq \frac{\epsilon(\varphi(x)+\omega) \varrho_{\varphi}}{1-\left(\frac{2 M_{f} T^{\alpha}}{\left(1-N_{f}\right) \Gamma(\alpha+1)}\right)}
$$

Using (21) and (22), for $x \in J$, we have

$$
\|s-z\|_{E} \leq \epsilon(\varphi(x)+\omega)\left[\frac{\left(\varrho_{\varphi}(1+k)+k\right)}{1-\left(\frac{2 M_{f} T^{\alpha}}{\left(1-N_{f}\right) \Gamma(\alpha+1)}+\frac{2 M_{f} n T^{\alpha}}{\left(1-N_{f}\right) \Gamma(\alpha+1)}+A_{I}^{*} n\right)}+\frac{\varrho_{\varphi}}{1-\left(\frac{2 M_{f} T^{\alpha}}{\left(1-N_{f}\right) \Gamma(\alpha+1)}\right)}\right] .
$$

Thus

$$
\|s-z\|_{E} \leq C_{2} \epsilon(\varphi(x)+\omega)
$$

where

$$
C_{2}=\left[\frac{\left(\varrho_{\varphi}(1+k)+k\right)}{1-\left(\frac{2 M_{f} T^{\alpha}}{\left(1-N_{f}\right) \Gamma(\alpha+1)}+\frac{2 M_{f} n T^{\alpha}}{\left(1-N_{f}\right) \Gamma(\alpha+1)}+A_{I}^{*} n\right)}+\frac{\varrho_{\varphi}}{1-\left(\frac{2 M_{f} T^{\alpha}}{\left(1-N_{f}\right) \Gamma(\alpha+1)}\right)}\right] .
$$

Therefore problem (1) is HUR stable.

\section{Applications}

\section{Example 1}

$$
\left\{\begin{array}{l}
{ }_{0}^{c} D_{x}^{\frac{1}{2}} z(x)=\frac{e^{-\pi x}}{15}+\frac{e^{-x}}{38+x^{2}}\left(\sin (|z(x)|)+z\left(\frac{1}{4} x\right)+\sin \left(\left|{ }_{0}^{c} D_{x} \frac{1}{2} z(x)\right|\right)\right) \\
x \in[0,1], \quad x \neq \frac{1}{3}, \quad k=1, \\
z(0)=0, \\
\Delta z\left(\frac{1}{3}\right)=\frac{1}{10} z\left(\frac{1}{3}\right),
\end{array}\right.
$$

where $\alpha=\frac{1}{2}, J_{0}=\left[0, \frac{1}{3}\right], J_{1}=\left(\frac{1}{3}, 1\right]$. 
Set

$$
f\left(x, z(x), z(m x), u_{z}(x)\right)=\frac{e^{-\pi x}}{15}+\frac{e^{-x}}{38+x^{2}}\left(\sin (|z(x)|)+z\left(\frac{1}{4} x\right)+\sin \left(\left|{ }_{0}^{c} D_{x}{ }^{\frac{1}{2}} z(x)\right|\right)\right)
$$

with $\alpha=\frac{1}{2}$ and $m=\frac{1}{4}$. It is clear that $f$ is a jointly continuous function.

Using $\left(H_{2}\right)$, for any $z, \bar{z} \in R$, we have

$$
\begin{aligned}
\left|f\left(x, z(x), z(m x),{ }_{0}^{c} D_{x}^{\alpha} z(x)\right)-f\left(x, \bar{z}(x), \bar{z}(m x),{ }_{0}^{c} D_{x}^{\alpha} \bar{z}(x)\right)\right| \\
\leq \frac{1}{19}|z(x)-\bar{z}(x)| \\
\quad+\frac{1}{38}\left|{ }_{0}^{c} D_{x} \frac{3}{2} z(x)-{ }_{0}^{c} D_{x} \frac{3}{2} \bar{z}(x)\right| .
\end{aligned}
$$

Hence $\left(H_{2}\right)$ holds with $M_{f}=\frac{1}{19}$ and $N_{f}=\frac{1}{38}$. Set

$$
I_{k}(v)=\frac{1}{10} v,
$$

where $v \in R$. Then for $v, \bar{v} \in R$ and $k=1$, we have

$$
\begin{aligned}
\left|I_{1}(v)-I_{1}(\bar{v})\right| & \leq\left|\frac{1}{10} v-\frac{1}{10} \bar{v}\right| \\
& \leq \frac{1}{10}|v-\bar{v}| .
\end{aligned}
$$

Hence $\left(A_{3}\right)$ holds with $A_{I}^{*}=\frac{1}{10}$.

Also, the condition

$$
\left(\frac{M_{f} T^{\alpha}}{\left(1-N_{f}\right) \Gamma(\alpha+1)}+\frac{M_{f} n T^{\alpha}}{\left(1-N_{f}\right) \Gamma(\alpha+1)}+A_{I}^{*} n\right)=0.169<1
$$

is satisfied with $T=1$ and $n=1$. Therefore by Theorem 3 problem (23) has a unique solution.

Let $\varphi(x)=x$ and $\omega=1$. Then for any $x \in[0,1]$, we have

$$
\begin{aligned}
I^{\frac{1}{2}} \varphi(x) & =\frac{1}{\Gamma\left(\frac{1}{2}\right)} \int_{0}^{x}(x-\tau)^{\frac{1}{2}-1} \tau d \tau \\
& \leq \frac{2 x}{\sqrt{\pi}} .
\end{aligned}
$$

We see that condition $\left(A_{8}\right)$ holds with $\varrho_{\varphi}=\frac{2}{\sqrt{\pi}}$. Applying 5, problem (23) is HUR stable.

\section{Conclusion}

Pantograph differential equations is a special type of delay differential equations with proportional delay terms. In this research work, we studied important aspects such as the existence theory and stability analysis to an IVP of pantograph implicit fractional differential equations with impulsive conditions. Using Schaefer's fixed point theorem, we derived a result of at least one solution to system (1), and applying the Banach contraction theorem, we obtained conditions for a unique solution to the mentioned problem. Similarly, using the Hyers-Ulam concept, we studied the stability of the considered problem. 


\section{Acknowledgements}

Ibrahim Mahariq and Bahaa Al-Sheikh would like to acknowledge College of Engineering and Technology at American University of the Middle East. Thabet Abdeljawad would like to thank Prince Sultan University for funding this work through research group Nonlinear Analysis Methods in Applied Mathematics (NAMAM), group number RG-DES-2017-01-17.

Funding

Not applicable.

\section{Availability of data and materials}

None.

\section{Competing interests}

The authors declare that they have no competing interests.

\section{Authors' contributions}

All authors have equal contribution in this manuscript. All authors read and approved the final manuscript.

\section{Author details}

'Department of Mathematics, University of Malakand, 18000, Chakdara, Dir(L), Khyber Pakhtunkhwa, Pakistan. ${ }^{2}$ College of Engineering and Technology, American University of the Middle East, Egaila, Kuwait. ${ }^{3}$ Department of Mathematics and General Sciences, Prince Sultan University, Riyadh, Saudi Arabia. ${ }^{4}$ Department of Medical Research, China Medical University, Taichung, 40402, Taiwan. ${ }^{5}$ Department of Computer Science and Information Engineering, Asia University, Taichung, Taiwan. ${ }^{6}$ Biomedical Systems and Medical Informatics Engineering, Yarmouk University, Irbid, Jordan.

\section{Publisher's Note}

Springer Nature remains neutral with regard to jurisdictional claims in published maps and institutional affiliations.

Received: 30 July 2020 Accepted: 5 January 2021 Published online: 19 January 2021

\section{References}

1. Yassen, M.F., Attiya, A.A., Agarwal, P.: Subordination and superordination properties for certain family of analytic functions associated with Mittag-Leffler function. Symmetry 12(10), 1724 (2020)

2. Fernandez, A.: An elliptic regularity theorem for fractional partial differential operators. Comput. Appl. Math. 37 5542-5553 (2018)

3. Agarwal, P., Deniz, S., Jain, S., Alderremy, A.A., Aly, S.: A new analysis of a partial differential equation arising in biology and population genetics via semi analytical techniques. Phys. A, Stat. Mech. Appl. 542, 122769 (2020)

4. Ali, A., Humaira, L., Shah, K.: Analytical solution of general Fisher's equation by using Laplace Adomian decomposition method. J. Pure Appl. Math. 2(3), 01 (2018)

5. Khalid, A., Naeem, M.N., Agarwal, P., Ghaffar, A., Ullah, Z., Jain, S.: Numerical approximation for the solution of linear sixth order boundary value problems by cubic B-spline. Adv. Differ. Equ. 2019(1), 492 (2019)

6. Kilbas, A.A., Srivastava, H., Trujillo, J.: Theory and Applications of Fractional Differential Equations. North-Holland Mathematics Studies., vol. 204. Elsevier, Amsterdam (2006)

7. Marks, R.J.I.I., Hall, M.W.: Differintegral interpolation from a bandlimited signals samples. IEEE Trans. Acoust. Speech Signal Process. 9(2), 872-877 (1981)

8. Torvik, P.J., Bagley, R.L.: On the appearance of the fractional derivative in the behavior of real materials. J. Appl. Mech. 51(2), 294-298 (1984)

9. Fernandez, A., Baleanu, D., Fokas, A.S.: Solving PDEs of fractional order using the unified transform method. Appl. Math. Comput. 339, 738-749 (2018)

10. Almeida, R., Bastos, N.R.O., Monteiro, M.T.T.: Modelling some real phenomena by fractional differential equations. Math. Methods Appl. Sci. 39(16), 4846-4855 (2016)

11. Caponetto, R., Dongola, G., Fortuna, L., Petras, I.: Fractional Order Systems. Modeling and Control Applications. World Scientific, River Edge (2010)

12. Kilbas, A.A., Marichev, O.I., Samko, S.G.: Fractional Integrals and Derivatives: Theory and Applications. Gordon \& Breach, Switzerland (1993)

13. Alderremy, A.A., Saad, K.M., Agarwal, P., Aly, S., Jain, S.: Certain new models of the multi space-fractional Gardner equation. Phys. A, Stat. Mech. Appl. 545, 123806 (2020)

14. Ali, A., Shah, K.: Ulam-Hyers stability analysis of a three-point boundary-value problem for fractional differential equations. Ukr. Mat. Zh. 72(2), 147-160 (2020)

15. Agarwal, P., Baltaeva, U., Alikulov, Y.: Solvability of the boundary-value problem for a linear loaded integro-differential equation in an infinite three-dimensional domain. Chaos Solitons Fractals 140, 110108 (2020)

16. Dong, J., Feng, Y., Jiang, J.: A note on implicit fractional differential equations. Math. Aterna 7(3), 261-267 (2017)

17. Abbas, S., Benchohra, M., Graef, J.R., Henderson, J.: Implicit Fractional Differential and Integral Equations: Existence and Stability, vol. 26. de Gruyter, Berlin (2018)

18. Tate, S., Dinde, H.T.: Boundary value problems for nonlinear implicit fractional differential equations. J. Nonlinear Anal. Appl. 2019, 29-40 (2019)

19. Ali, A., Shah, K., Jarad, F., Gupta, V., Abdeljawad, T.: Existence and stability analysis to a coupled system of implicit type impulsive boundary value problems of fractional-order differential equations. Adv. Differ. Equ. 2019(101), 1 (2019)

20. Tian, Y., Bai, Z.: Existence results for the three-point impulsive boundary value problem involving fractional differential equations. Comput. Math. Appl. 59(8), 2601-2609 (2010) 
21. Ali, A., Shah, K., Baleanu, D.: Ulam stability results to a class of nonlinear implicit boundary value problems of impulsive fractional differential equations. Adv. Differ. Equ. 2019(5), 1 (2019)

22. Wang, J., Yang, Y., Wei, W.: Nonlocal impulsive problems for fractional differential equations with time-varying generating operators in Banach spaces. Opusc. Math. 30(3), 361-381 (2010)

23. Andronov, A., Witt, A., Haykin, S.: Oscilation Theory. Nauka, Moscow (1981)

24. Ali, A. Rabiei, F. Shah, K.: On Ulam's type stability for a class of impulsive fractional differential equations with nonlinear integral boundary conditions. J. Nonlinear Sci. Appl. 10(9), 4760-4775 (2017)

25. Zavalishchin, S., Sesekin, A.: Impulsive Processes: Models and Applications. Nauka, Moscow (1991)

26. Vinodkumar, A., Malar, K., Gowrisankar, M., Mohankumar, P.: Existence, uniqueness and stability of random impulsive fractional differential equations. Acta Math. Sci. 36(2), 428-442 (2016)

27. Pappalardo, C.M., De Simone, M.C., Guida, D.: Multibody modeling and nonlinear control of the pantograph/catenary system. Arch. Appl. Mech. 89(8), 1589-1626 (2019)

28. Li, D., Zhang, C.: Long time numerical behaviors of fractional pantograph equations. Math. Comput. Simul. 172 244-257 (2020)

29. Karimi Vanani, S., Sedighi Hafshejani, J., Soleymani, F., Khan, M.: On the numerical solution of generalized pantograph equation. World Appl. Sci. J. 13(12), 2531-2535 (2011)

30. Bogachev, L., Derfel, G., Molchanov, S., Ochendon, J.: On bounded solutions of the balanced generalized pantograph equation. In: Chow, P.-L., Yin, G., Mordukhovich, B. (eds.) Topics in Stochastic Analysis and Nonparametric Estimation. The IMA Volumes in Mathematics and Its Applications, vol. 145, pp. 29-49. Springer, New York (2008)

31. Chamekh, M., Elzaki, T.M., Brik, N.: Semianalytical solution for some proportional delay differential equations. SN Appl. Sci. 1, 148 (2019)

32. Podlubny, l.: Frictional Differential Equations. Academic Press, San Diego (1999)

33. Cabada, A., Wang, G.: Positive solutions of nonlinear fractional differential equations with integral boundary value conditions. J. Math. Anal. Appl. 389(1), 403-411 (2013)

34. Rus, I.A.: Ulam stabilities of ordinary differential equations in a Banach space. Carpath. J. Math. 26, 103-107 (2010)

35. Granas, A., Dugundji, J.: Fixed Point Theory. Springer, Berlin (2013)

\section{Submit your manuscript to a SpringerOpen ${ }^{\circ}$ journal and benefit from:}

- Convenient online submission

- Rigorous peer review

- Open access: articles freely available online

- High visibility within the field

- Retaining the copyright to your article

Submit your next manuscript at $\boldsymbol{\nabla}$ springeropen.com 